with ST-3672 $(n=22)$ predominant. At the bivariate level, infection with ST-3672 was associated with younger age $(62 \%$ of those infected were 15-19 years old, $\mathrm{p}=0.002)$, and chlamydia co-infection $(67 \%$ vs $37 \%, \mathrm{p}=0.012)$. In multivariable analysis, age group remained significant, while an interaction between inner-core residency and chlamydia co-infection was detected. Case-contact networks were highly-fragmented, consisting mainly of dyads and triads. Of 85 components, the largest component included 6 nodes, while 61\% were dyads. CUG testing indicated in-degree centralization was lower than expected $(\mathrm{p}<0.05)$. Genotyping combined with case-contact data increased the potential size and geographic reach of each component. Of potential components found after incorporating subtypes, 32\% (10/33) were dyadic, with the largest component consisting of 45 nodes.

Conclusion Molecular data revealed connections that were not apparent from case-contact investigations alone, leading to more cases potentially linked together, and over a wider geographic area. A handful of subtypes were responsible for the majority of infections. Early identification of dominant subtypes may potentially curtail transmission of NG.

Disclosure No significant relationships.

\section{P688 RECENT INCREASES IN RATES OF GONORRHEA IN TORONTO, ONTARIO, 2012-2018}

Dana Al-Bargash*. Toronto Public Health, Communicable Disease Surveillance Unit Communicable Disease Control, Toronto, Canada

10.1136/sextrans-2019-sti.754

Background In Toronto, gonorrhea is the second most commonly reported sexually transmitted infection, after chlamydia. From 2000 to 2012, rates of gonorrhea in Toronto were stable, ranging from $56 / 100,000$ to $72 / 100,000$. However, rates started to rise in 2013. In 2018, rates increased by $37 \%$ from 2017, the largest observed annual increase since 2000, reaching a high of $158 / 100,000$. This study aimed to describe gonorrhea trends in Toronto between 2012 and 2018 .

Methods Data for gonorrhea cases reported between 2012 and 2018 were extracted from the integrated Public Health Information System on January 29 2019. Analyses were conducted in SAS 9.4.

Results In 2018, 4,549 gonorrhea cases were reported in Toronto, $135 \%$ higher than 1,939 cases $(71 / 100,000)$ reported in 2012. The increase was driven by a rise in reports among males, increasing by $192 \%$ while females increased by $24 \%$; males comprised $81 \%$ of cases in 2018. Males most commonly reported engaging in sex with men (MSM), and the proportion with this risk factor increased from 55\% in 2012 to $69 \%$ in 2018 . Conversely, the proportion of males reporting sex with women declined from $25 \%$ in 2012 to $17 \%$ in 2018. Females in 2018 most commonly reported not using a condom $(77 \%)$ in the last sexual encounter, slightly higher than 2012 (71\%). In 2018, 38\% of cases (44\% of males, 9\% of females) had rectum and/or pharyngeal gonorrhea, higher than $20 \%$ of cases in 2017.

Conclusion The rising rates in gonorrhea, particularly among MSM, may be due to changes in screening guidelines in 2013 that included extragenital screening of gonorrhea. In April 2018, both rectal and pharyngeal specimens were approved for Nucleic Acid Amplification Testing in Ontario, potentially playing a role in the additional increase in 2018. This study demonstrates that it is important that physicians continue to screen for extragenital gonorrhea among MSM.

Disclosure No significant relationships.

\section{P689 TURNING GONORRHEA AGAINST HIV: LATENT HIV 'SHOCK-AND-KILL' USING A GONOCOCCAL-DERIVED METABOLITE}

Scott Gray-Owen*, Furkan Guvenc. University of Toronto, Molecular Genetics, Toronto, Canada

\subsection{6/sextrans-2019-sti.755}

Background Clinical studies have long indicated that a pathological synergy exists between Neisseria gonorrhoeaeand HIV, with gonococcal infection increasing HIV transmission between HIV serodiscordant sexual partners. In trying to understand this association, we discovered that $N$. gonorrhoeae liberate a small molecule that stimulates HIV replication from latently infected CD4 + T cells. This led to our discovery that heptose phosphate (HP)-containing metabolites, 7-carbon phospho-sugars not produced by animals, serve as a molecular cue that bacteria are present in the tissues and elicit an NF- $\mathrm{BB}$-dependent transcriptional response. Based upon these observations, this study aims to test the hypothesis that HP can function both to (i) drive the virus from latency and (ii) stimulate the antiviral response to work in synergy with available highly active antiretroviral therapies to cure HIV infection.

Methods We have used a combination of cell line and primary human leukocyte-based models to test the effect of natural and synthetic analogues of HP to stimulate HIV from latency, both alone and in combination with potential latency reversing agents, and to understand their effect of HPs on different leukocytic populations that have potential to either promote or inhibit HIV infection.

Results We show that HP has a superior combination of HIV latency reversal without toxicity often evident with conventional LRAs, and HP activity synergizes with other LRAs such that these can be administered at lower concentrations. Finally, we observed that HP stimulates primary human leukocytic responses with anti-viral potential.

Conclusion Our findings suggest that HP-based agonists are a novel LRA capable of both driving HIV from latency and stimulating immune responses so as to help control the infection. By virtue of its synergy with other LRAs and clinically available anti-retroviral agents, this represents an enticing new avenue in ongoing efforts to develop a cure for established HIV infection.

Disclosure No significant relationships.

\section{P690 ESTABLISHMENT OF THE GONORRHEA MOUSE MODEL FOR PRE-CLINICAL TESTING OF ANTIBIOTICS THAT FOLLOW THE PK DRIVER FAUC/MIC}

Kristie Connolly*, Lenise Soileau, Ann Jerse. F. Edward Hebert School of Medicine, Uniformed Services of the Health Sciences, Microbiology and Immunology, Bethesda, USA

\subsection{6/sextrans-2019-sti.756}

Background New antibiotics for gonorrhea are needed due to the emergence of resistance to extended-spectrum cephalosporins in Neisseria gonorrhoeae $(\mathrm{Ng})$. We recently established the $17 \beta$-estradiol mouse model of gonococcal lower genital tract 